\title{
On the Use of Lattice-Boltzmann Model for Simulating Lid-Driven Cavity Flows of Strain-hardening Fluids
}

\author{
Mohammad-Mehdi HedAYAT, Mohammad-Hossein BorgHEI, Abbas FAKHARI, and Kayvan SADEGHY* \\ Department of Mechanical Engineering, College of Engineering, \\ University of Tehran, Tehran, Iran P.O. Box: 11155 - 4563
}

(Received: June 21, 2010)

\begin{abstract}
The effect of a fluid's strain-hardening behavior is investigated on the flow kinematics inside a two-dimensional square cavity. A special form of the Generalized Newtonian Fluid model (referred to as Pinho model) is used in which the strain-hardening behavior of the fluid is accounted for by incorporating a strain-rate term in the power-law viscosity function. Use will be made of the incompressible Lattice Boltzmann Model (LBM) to numerically solve the governing equations. Converged results could be obtained at Reynolds numbers up to $\mathrm{Re}=5000$ showing that the strain-hardening behavior has indeed a strong effect on the flow kinematics. The size of the corner vortices is predicted to decrease by an increase in the strain-hardening behavior of the fluid. It is also predicted that the streamline pattern becomes quite symmetric by an increase in the fluid's strain-hardening behavior.
\end{abstract}

Key Words: Lid-driven cavity flow / Lattice boltzmann / Multiple relaxation time / Generalized newtonian fluid model

\section{INTRODUCTION}

Computational methods play a key role in modern fluid dynamics. Currently a large number of complex problems in fluid mechanics can accurately be solved using numerical methods. The success of computational methods in fluid mechanics is often credited to the advent of high-performance hardware. But, credit should also be given to the development of more efficient algorithms and numerical methods. One can mention, for example, the Lattice Boltzmann Method (LBM) ${ }^{1)}$ which is the new addition to the list of numerical methods comprising Finite Difference Method (FDM), Finite Volume Method (FVM), Finite Element Method (FEM), Boundary Element Method (BEM), and Spectral Method (SM). The LBM appears to be particularly suitable for tackling problems in non-Newtonian fluid mechanics because the rate-ofdeformation tensor is computed locally thereby eliminating the need for computing space derivatives of the velocity field. ${ }^{2,3)}$ This notion has beautifully been demonstrated by Aharonov and Rothman ${ }^{4}$ for purely-viscous non-Newtonian fluids obeying the power-law model. In subsequent works, LBM has successfully been used to simulate the flow of several other non-Newtonian fluid models in a variety of flow configurations including lid-driven cavity. ${ }^{5-25)}$

* to whom correspondence should be addressed.
Among different flow geometries used in the above studies, the case of lid-driven cavity flow is of particular interest in that it covers a wide range of flow phenomena encompassing recirculation, singularity, instability, and transient behavior even for Newtonian fluids. ${ }^{26)}$ As expected, this already complicated picture becomes even more complicated if the fluid is non-Newtonian. That is to say that, for these fluids further complications may arise as a result of relaxation effects, normal stress effects, shear-thinning/shear-thickening effects, and strain-hardening effects. Thus, it should not be surprising that interpretation of experimental data obtained, say, for polymer solutions in cavity flow is not an easy task. For example, it is not clear to what extent a drop in the degree of mixing, as observed in cavity flow of polymer solutions, is due to the strain-hardening effects because the picture is marred by simultaneous normal stress and/or relaxation effects. ${ }^{27-29)}$

Fortunately, theoretical methods can shed some light on issues like these by addressing each effect separately, say through the use of simplistic fluid models. This is perhaps why there are so many works in the literature relying on purelyviscous fluid models (e.g., power-law model) regardless of the fact that the same mechanism which gives rise to shearthinning in polymeric liquids is also responsible for their elasticity. With this in mind, in the present work we are going to address the sole effects of a fluid's strain-hardening behavior on cavity flow using a simple fluid model which excludes 
complications arising from normal stress and/or relaxation effects. To that end, use will be made of a special form of the Generalized Newtonian Fluid (GNF) model modified in such a way that it can accommodate strain-rate dependent viscosity through a power-law term. To better handle the corner singularities, the governing equations will be solved using the Lattice-Boltzmann method. To the best of our knowledge, this robust numerical method has not previously been applied to the cavity flows of purely strain-hardening fluids.

\section{THEORETICAL FORMULATIONS}

Figure 1 shows a schematic representation of a twodimensional lid-driven square cavity having an infinite width in the $z$-direction. The fluid inside the cavity is set into motion by the movement of the upper lid with a constant speed $U_{0}$. Under steady conditions, the equations governing the flow of any incompressible fluid are,

$$
\begin{aligned}
& \rho\left(u \frac{\partial u}{\partial x}+v \frac{\partial u}{\partial y}\right)=-\frac{\partial p}{\partial x}+\frac{\partial \tau_{x x}}{\partial x}+\frac{\partial \tau_{x y}}{\partial y} \\
& \rho\left(u \frac{\partial v}{\partial x}+v \frac{\partial v}{\partial y}\right)=-\frac{\partial p}{\partial y}+\frac{\partial \tau_{y x}}{\partial x}+\frac{\partial \tau_{y y}}{\partial y} \\
& \frac{\partial u}{\partial x}+\frac{\partial v}{\partial y}=0
\end{aligned}
$$

where the boundary conditions are the no-slip and nopenetration at the rigid walls. As to the stress components, in the present work, it is assumed that the fluid of interest obeys Pinho's model. This rheological model is, in fact, a variation of the Generalized Newtonian Fluid (GNF) model modified such that it can represent strain-hardening fluids. The Generalized Newtonian Fluid (GNF) model is an inelastic rheological model in which the stress tensor is written $\mathrm{as}^{30}$ :

$$
\tau_{\mathrm{ij}}=2 \mu \mathbf{d}=\mu\left(\frac{\partial \mathrm{u}_{\mathrm{i}}}{\partial \mathrm{x}_{\mathrm{j}}}+\frac{\partial \mathrm{u}_{\mathrm{j}}}{\partial \mathrm{x}_{\mathrm{i}}}\right)
$$

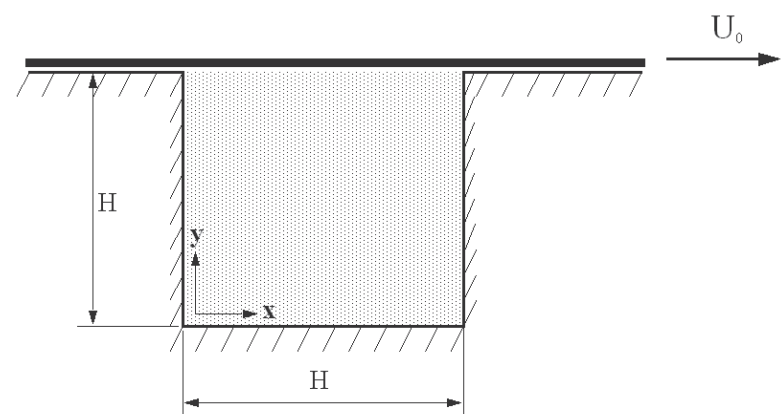

Fig. 1. Sketch of the two-dimensional lid-driven cavity. where $\mathbf{d}$ is the rate-of-deformation tensor, and $\mu$ is the absolute viscosity of the fluid. Unlike Newtonian fluids, for which $\mu$ is a constant, for a fluid obeying the GNF model the viscosity is allowed to be shear-dependent. Having said this, it should be conceded that the model cannot predict relaxation effects nor can it predict normal stress differences in shear and/or extensional flows. $^{30)}$ Thus, it is good only for inelastic fluids. Pinho modified the model in such a way that the viscosity function depends on the rate of extension in addition to the rate of shear, i.e., $\mu(\dot{\gamma}, \dot{\varepsilon})$. In Pinho's variation of the GNF model $^{31,32)}$, the viscosity is written as:

$$
\mu=\left(\mathrm{K}_{\mathrm{v}}|\dot{\gamma}|^{(\mathrm{n}-1)}\right) \cdot\left(\mathrm{K}_{\mathrm{e}}|\dot{\varepsilon}|^{(\mathrm{p}-1)}\right)
$$

where $\dot{\gamma}$ and $\dot{\varepsilon}$ are related to the invariants of $\mathbf{d}$ by the following relationships (see the Appendix A for more details):

$$
\begin{aligned}
& \dot{\gamma}=\sqrt{-4 \mathrm{II}_{\mathrm{d}}}=\sqrt{2 \mathrm{~d}_{\mathrm{ij}} \mathrm{d}_{\mathrm{ij}}} \\
& \dot{\varepsilon}=\frac{6 \operatorname{det} \mathbf{d}}{\operatorname{tr} \mathbf{d}^{2}}=\frac{2 \operatorname{tr} \mathbf{d}^{3}}{\operatorname{tr} \mathbf{d}^{2}}=\frac{2\left(\mathrm{~d}_{\mathrm{ik}} \mathrm{d}_{\mathrm{kj}}\right) \mathrm{d}_{\mathrm{ij}}}{\mathrm{d}_{\mathrm{ij}} \mathrm{d}_{\mathrm{ij}}}
\end{aligned}
$$

It needs to be mentioned that the model parameters (i.e., $K_{\mathrm{v}}$, $K_{\mathrm{e}}, n$ and $p$ ) are material properties which must be determined using rheological data. It is also to be noted that this simple rheological model can represent Newtonian fluids by simply setting $n=p=1$. Interestingly, it can represent shear-thinning and shear-thickening liquids by allowing $n$ to differ from one (for any given $p$ ). Similarly, it can represent strain-softening and strain-hardening fluids by allowing $p$ to differ from one (for any given $n$ ). As shown schematically in Fig. 2, for the model to predict strain-hardening behavior of polymeric liquids, the exponent $p$ should be larger than one. Figure 2 also shows that the fluid becomes increasingly more strain-

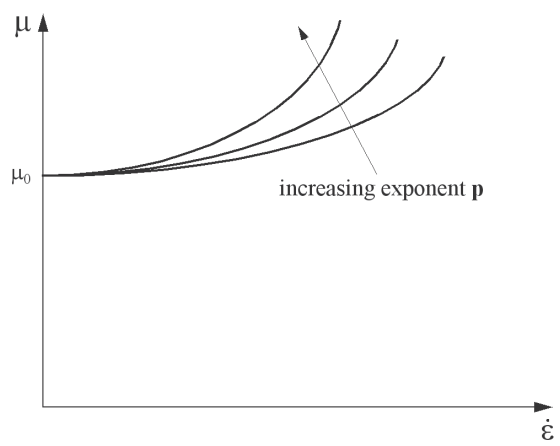

Fig. 2. Schematic showing the effect of the exponent $p$ (see Eq. 3) on the viscosity of a fluid. 
hardening the higher the power-law exponent $p$. It needs to be mentioned that in this figure $\mu_{0}$ is the fluid's viscosity at zero deformation rate.

\section{METHOD OF SOLUTION}

As mentioned above, in the present work we are going to rely on the Lattice-Boltzmann method for solving the equations of motion. In the incompressible limit, the generalized Boltzmann equation is written $\mathrm{as}^{33 \text { ), }}$

$$
\frac{\mathrm{Dg}}{\mathrm{Dt}}=\frac{\partial \mathrm{g}}{\partial \mathrm{t}}+\xi \cdot \nabla \mathrm{g}=-\mathrm{S}\left(\mathrm{g}-\mathrm{g}^{\mathrm{eq}}\right)
$$

where $g$ is the particle distribution function, $t$ stands for the time, $\xi$ is the microscopic velocity, $g^{\text {eq }}$ is the equilibrium distribution function in the phase space, and $\mathbf{S}$ is the general relaxation matrix. The following macroscopic mass and momentum equations (i.e., the Navier-Stokes equations) can be recovered by a proper Chapman-Enskog analysis ${ }^{33)}$ :

$$
\begin{aligned}
& \frac{\partial \rho}{\partial \mathrm{t}}+\nabla \cdot(\rho \mathbf{u})=0 \\
& \rho \frac{D \mathbf{u}}{D t}=-\nabla p+\nabla \cdot \Pi
\end{aligned}
$$

in which $\Pi=\mu\left[\nabla_{\mathrm{u}}+\left(\nabla_{\mathrm{u}}\right)^{\mathrm{T}}\right]$ is the viscous stress tensor, $\rho$ is the density, $\mathbf{u}$ is the macroscopic velocity, $\mu$ is the dynamic viscosity, and $p$ is the pressure. In the present work, to discretize Eq. 7 use will be made of the D2Q9 lattice (see Appendix B) in which the microscopic velocities are defined by ${ }^{33)}$,

$$
\mathrm{e}_{\alpha}=\left\{\begin{array}{ccc}
(0,0), & \alpha=0 \\
\mathrm{c}(\cos \theta, \sin \theta), & \theta=\frac{(\alpha-1) \pi}{2}, & \alpha=1,2,3,4 \\
\sqrt{2} \mathrm{c}(\cos \theta, \sin \theta), & \theta=\frac{(\alpha-5) \pi}{2}+\frac{\pi}{4}, & \alpha=5,6,7,8
\end{array}\right.
$$

where $c=\delta x / \delta t$ is the lattice speed. Adopting a summation convention for repeated subscript indices, the discretized form of the Boltzmann equations may be written as

$$
\mathrm{g}_{\alpha}\left(\mathrm{x}+\mathrm{e}_{\alpha} \delta_{\mathrm{t}}, \mathrm{t}+\delta_{\mathrm{t}}\right)=\mathrm{g}_{\alpha}(\mathrm{x}, \mathrm{t})-\mathrm{S}_{\alpha 1}\left(\mathrm{~g}_{1}-\mathrm{g}_{1}^{\mathrm{eq}}\right)
$$

where $\mathbf{x}$ is the position vector. The above equations can be mapped onto the moment space by multiplying it by an invertible transformation matrix $\mathbf{M}$,

$$
\hat{\mathrm{g}}_{\alpha}\left(\mathrm{x}+\mathrm{e}_{\alpha} \delta_{\mathrm{t}}, \mathrm{t}+\delta_{\mathrm{t}}\right)=\hat{\mathrm{g}}_{\alpha}(\mathrm{x}, \mathrm{t})-\hat{\mathrm{S}}_{\alpha \mathrm{i}}\left(\hat{\mathrm{g}}_{\mathrm{i}}-\hat{\mathrm{g}}_{\mathrm{i}}^{\mathrm{eq}}\right)
$$

where,

$$
\hat{g}=\mathbf{M} g \quad ; \quad \hat{g}^{e q}=\mathbf{M} g^{e q}
$$

The transformation matrix is explicitly given by

$$
\mathbf{M}=\left[\begin{array}{ccccccccc}
1 & 1 & 1 & 1 & 1 & 1 & 1 & 1 & 1 \\
-4 & -1 & -1 & -1 & -1 & 2 & 2 & 2 & 2 \\
4 & -2 & -2 & -2 & -2 & 1 & 1 & 1 & 1 \\
0 & 1 & 0 & -1 & 0 & 1 & -1 & -1 & 1 \\
0 & -2 & 0 & 2 & 0 & 1 & -1 & -1 & 1 \\
0 & 0 & 1 & 0 & -1 & 1 & 1 & -1 & -1 \\
0 & 0 & -2 & 0 & 2 & 1 & 1 & -1 & -1 \\
0 & 1 & -1 & 1 & -1 & 0 & 0 & 0 & 0 \\
0 & 0 & 0 & 0 & 0 & 1 & -1 & 1 & -1
\end{array}\right]
$$

The diagonal relaxation matrix $\hat{\mathbf{S}}$ in moment space reads as

$$
\hat{\mathrm{S}}=\mathrm{MSM}^{-1}=\operatorname{diag}\left(\mathrm{s}_{1}, \mathrm{~s}_{2}, \mathrm{~s}_{3}, \mathrm{~s}_{4}, \mathrm{~s}_{5}, \mathrm{~s}_{6}, \mathrm{~s}_{7}, \mathrm{~s}_{8}, \mathrm{~s}_{9}\right)
$$

In the BGK model, the collision operator $\mathbf{S}$ is a diagonal matrix whose elements are $1 / \tau$, where $\tau$ is the dimensionless relaxation time. But in the MRT model, the collision operator is a full matrix. Here, we choose $s_{1}=s_{4}=s_{6}=0$ (based on the conservation laws), and arbitrarily set $s_{2}=1.1$, $s_{3}=1.4, s_{5}=s_{7}=1.7, s_{8}=s_{9}=1 / \tau$ as the relaxation rates. The equilibrium distributions, in the moment space, are given by:

$$
\hat{g}^{e q}=\rho R T\left[\begin{array}{c}
1 \\
-2+3 u^{2} \\
1-3 u^{2} \\
u_{x} \\
-u_{x} \\
u_{y} \\
-u_{y} \\
u_{x}^{2}-u_{y}^{2} \\
u_{x} u_{y}
\end{array}\right]
$$

where $R$ is the gas constant, $T$ is the temperature, and $u_{\mathrm{x}}$ and $u_{\mathrm{y}}$ are the $x$ - and $y$-components of velocity, respectively. The macroscopic variables shall be calculated after the streaming step (see Appendix B) via the following relations

$$
\begin{aligned}
& p=\sum_{\alpha=0}^{8} \bar{g}_{\alpha} \\
& \rho R T \mathbf{u}=\sum_{\alpha=1}^{8} \bar{g}_{\alpha} \mathbf{e}_{\alpha} \\
& \rho R T \mathbf{u}=\sum_{\alpha=1}^{8} \bar{g}_{\alpha} \mathbf{e}_{\alpha}
\end{aligned}
$$




\section{RESULTS AND DISCUSSIONS}

In this section, numerical results obtained using the numerical method described above will be presented for Pinho's model. Before so doing, the LBM code developed in this work had to be verified first. To that end, we have decided to check the efficiency of our LBM code by comparing its output for Newtonian fluids with the FDM results of Ghia et al. $^{26)}$ Table I compares the two sets of results obtained using the same 129 x 129 grid for both velocity components at the midpoint of the cavity. As can be seen in this Table, a good comparison exists between these two methods of solution thus verifying the LBM code developed in this work.

Having validated the code, we are now at a stage to present our numerical results for cavity flow of Pinho's fluid. We are going to address the effect of $p$ and $n$ on the velocity profiles and streamline pattern inside the cavity although, as previously mentioned, our main interest in this work lies in investigating the effect of $p$. We have been able to obtain numerical results at Reynolds numbers as high as 5000. It needs to be mentioned that in the present work the Reynolds number is defined by,

$$
\mathrm{Re}=\frac{\rho H \mathrm{H}_{0}}{\mu_{\mathrm{r}}}
$$

where $\mu_{\mathrm{r}}$ is the reference viscosity. Since power-law fluids lack a zero-shear viscosity, in the present work we have decided to use $\mu_{\mathrm{r}}=\mathrm{K}_{\mathrm{v}} \mathrm{K}_{\mathrm{e}}$ as the reference viscosity. Based on Eq. 3, one can conclude that the reference viscosity used in this work is in fact the viscosity of a Pinho fluid measured at $\dot{\gamma}=\dot{\varepsilon}=1 \mathrm{~s}^{-1}$. Using this definition for the Reynolds number, Fig. 3 presents typical numerical results obtained at $\mathrm{Re}=$ 1000. This figure shows the effect of the power-law exponent $p$ on the velocity components $\mathrm{U}_{\mathrm{x}}$ and $\mathrm{U}_{\mathrm{y}}$ (made dimensionless using $\mathrm{U}_{0}$ ) in the vertical and horizontal mid-planes of the cavity. For comparison purposes, this figure also includes Ghia et $\mathrm{al}^{20)}$ data obtained using the finite differences method (FDM) for Newtonian fluids (i.e., for $n=p=1$ ). The strong influence of $p$ on the velocity profiles is evident in this figure.

Table I. A comparison between the results obtained in this work using LBM with Ghia's ${ }^{26)}$ benchmark results obtained using FDM for the $u$ - and $v$-velocity components at the midpoint of the cavity.

\begin{tabular}{ccccccc}
\hline \multirow{2}{*}{ Reference } & \multicolumn{6}{c}{ Re } \\
\cline { 2 - 7 } & \multicolumn{2}{c}{100} & \multicolumn{2}{c}{400} & \multicolumn{2}{c}{1000} \\
\cline { 2 - 7 } & $\mathrm{u}_{\mathrm{m}}$ & $\mathrm{V}_{\mathrm{m}}$ & $\mathrm{u}_{\mathrm{m}}$ & $\mathrm{V}_{\mathrm{m}}$ & $\mathrm{u}_{\mathrm{m}}$ & $\mathrm{V}_{\mathrm{m}}$ \\
\hline Present work & -0.20621 & 0.05718 & -0.11550 & 0.05202 & -0.06581 & 0.02631 \\
${\text { Ghia et } \mathrm{al}^{26)}}$ & -0.20581 & 0.05454 & -0.11477 & 0.05186 & -0.06080 & 0.02526 \\
\hline
\end{tabular}

To better understand the effect of $p$ on the flow kinematics inside the cavity, in Fig. 4 we have plotted its effect on the streamline pattern at $\operatorname{Re}=1000$. As can be seen in this figure, an increase in the strain-hardening behavior of the fluid (i.e., an increase in $p$ ) strongly affects the vortex structure inside the cavity. Interestingly, the two corner vortices are predicted to diminish in size by an increase in the fluid's strain-hardening behavior. This prediction has the implication that mixing becomes weaker for strain-hardening fluids. This prediction is in line with experimental observations made by Leong and Ottino ${ }^{27)}$ and has the implication that the level of mixing in cavity flow is strongly affected by the strain-hardening behavior of polymer solutions. The effect of strain-hardening on vortex structure can best be seen in Fig. 5 obtained at Re $=3200$. At this particular Reynolds number a corner vortex is known to form below the lid (say, at its upper-left corner) for Newtonian fluids. ${ }^{26)}$ As can be seen in this figure, this inertia vortex is completely suppressed by an increase in the strain-hardening behavior of the fluid implying that another vortex of an opposite sign has been generated weakening this

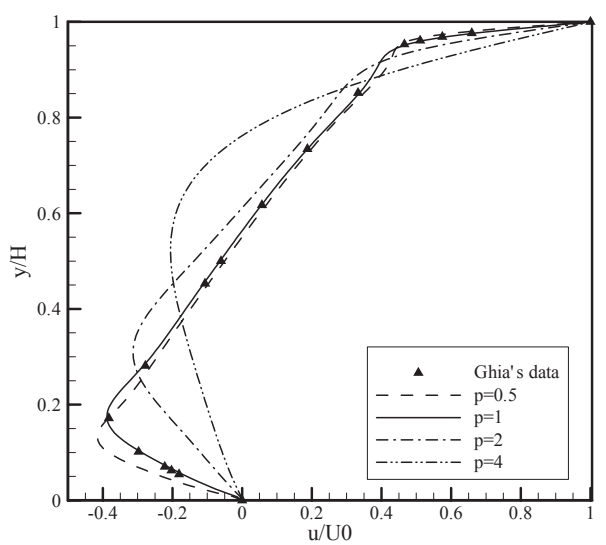

(a)

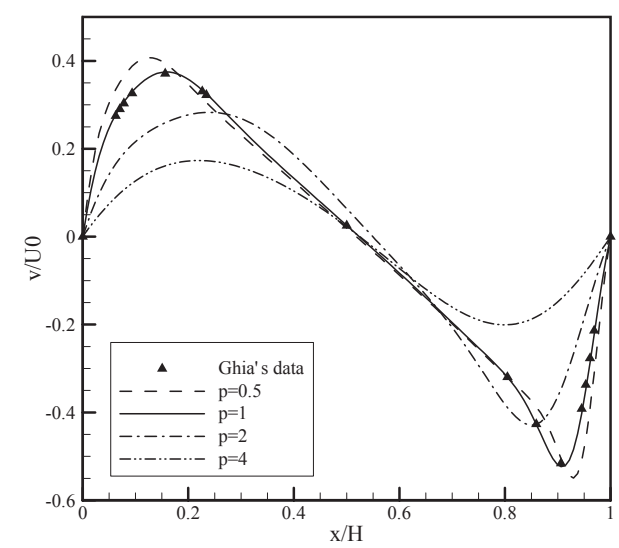

(b)

Fig. 3. Dimensionless velocity profiles along vertical and horizontal midplanes showing the effect of the power-law exponent $p$ at $\mathrm{Re}=$ 1000; (a) x-component velocity $\mathrm{U}_{\mathrm{x}}$, (b) y-component velocity $\mathrm{U}_{\mathrm{y}}$. 
vortex. It is also interesting to note that (see Figs. 4 and 5) by an increase in $p$, the geometric center of the core vortex shifts upwards and slightly to the right (i.e., in the direction of the lid motion).

Having investigated the effect of $p$ on the velocity profiles and streamline pattern, we have decided to investigate the effect of the power-law exponent $n$ on the flow. The idea was to see if similar effects can be obtained by varying $n$ instead of $p$. Figure 6 shows that, like $p$, the power-law exponent $n$ has a strong effect on the velocity profiles. But, what seems rather puzzling, at least at first sight, is the notion that an increase in $n$ has an effect opposite to that caused by a decrease in $p$ (cf. Figs. 3 and 6). This prediction seems rather peculiar realizing the fact that by an increase in $n$ the fluid becomes progressively more and more shear-thickening whereas by a decrease in $p$ it becomes more and more strain-softening.

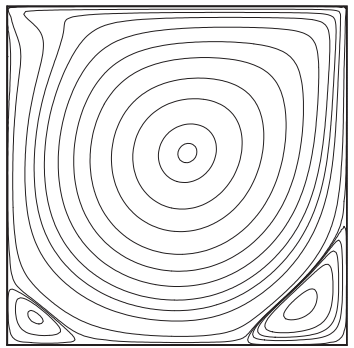

(a)

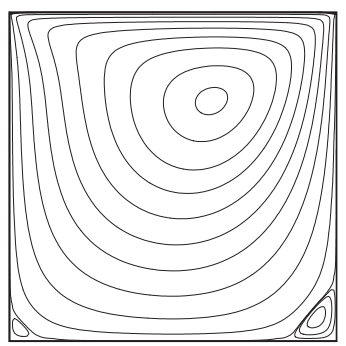

(c)

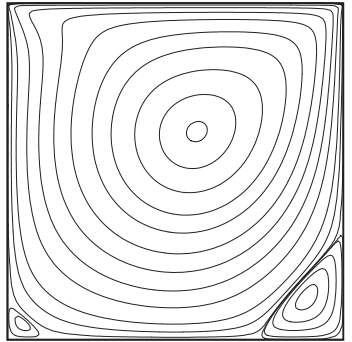

(b)

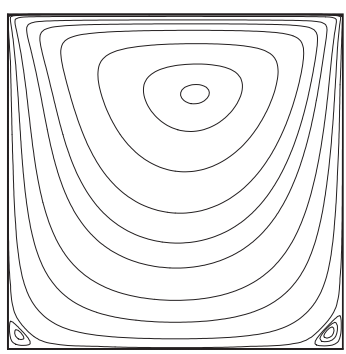

(d)
Fig. 4. Streamline pattern for Pinho's fluid obtained for $\mathrm{n}=1$ at $\mathrm{Re}=$ 1000: a) $\mathrm{p}=1$; b) $\mathrm{p}=2$; c) $\mathrm{p}=3$; d) $\mathrm{p}=4$.

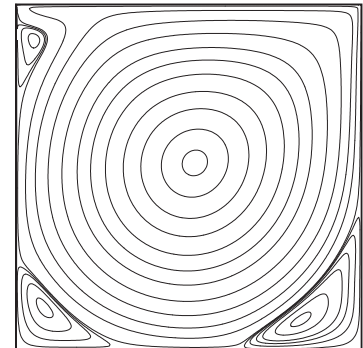

(a)

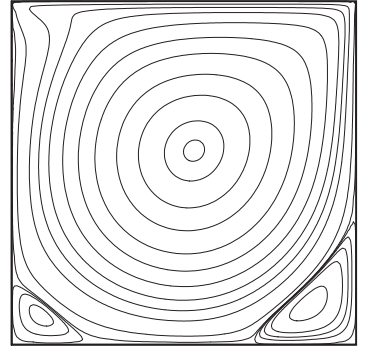

(b)
Fig. 5. Streamline pattern for Pinho's fluid at $\operatorname{Re}=3200$ : a) $n=p=1$; b) $\mathrm{n}=1, \mathrm{p}=2$.
The clue to this seemingly intriguing prediction lies in the magnitude of the absolute value of (dimensionless) shear and strain rates. That is, we have checked the magnitude of the shear and strain rates and realized that at the mid-plane of the cavity, the absolute value of the shear rate is less than one whereas the absolute value of the strain rate is larger than one. Since the shear rate is raised to a power of (see Eq. 3) one can conclude that the viscosity should decrease by an increase in $n$ provided that $n$ is larger than one (i.e., for shear-thickening fluids).

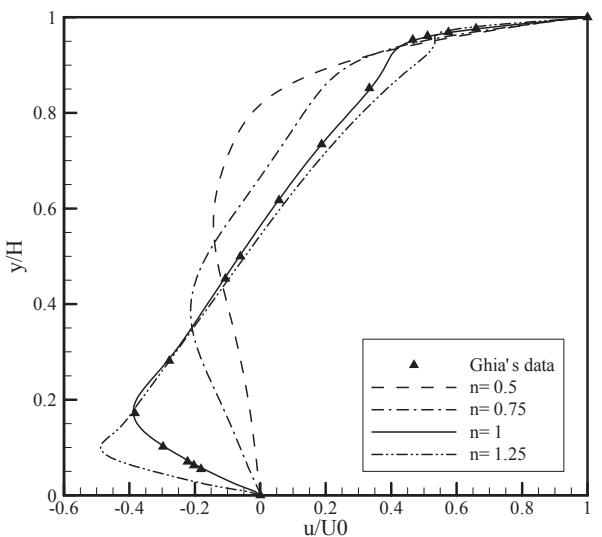

(a)

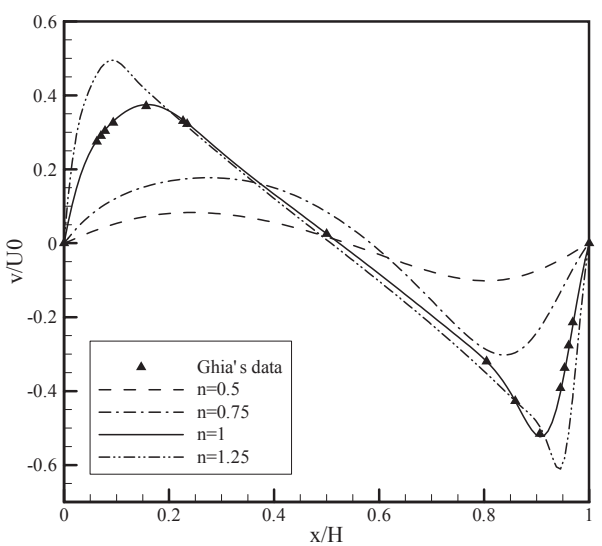

(b)

Fig. 6. Dimensionless velocity profiles along vertical and horizontal midplanes showing the effect of the exponent $n$ at $\mathrm{Re}=1000$ (a) x-component velocity $U_{x}$, (b) y-component velocity $U_{y}$.

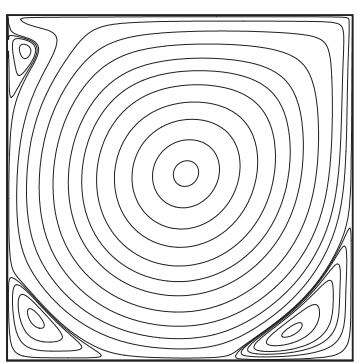

(a)

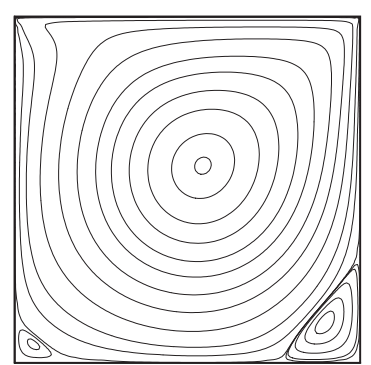

(b)
Fig. 7. Streamline pattern for Pinho's fluid at $R e=3200$; (a) $n=p=1$; (b) $\mathrm{n}=0.75, \mathrm{p}=1$. 


\section{CONCLUDING REMARKS}

Based on the results obtained in the present work it can be concluded that Pinho's model is well capable of predicting, at least qualitatively, the effects of a fluid's strain-hardening behavior on flow kinematics inside a square cavity. Numerical results obtained using the Lattice Boltzmann Method show that strain-hardening strongly affects the vortex structure inside the cavity. An increase in the degree of the strain-hardening behavior is predicted to shrink corner vortices giving rise to a more symmetric vortex structure within the cavity. The work also demonstrates that in cavity flow simulations, the behavior of strain-thickening fluids is qualitatively the same as the behavior of shear-thinning fluids. This rather unexpected result was attributed to the relative magnitudes of strain rate and shear rate in the mid-plane of the cavity.

\section{Acknowledgement}

This work is dedicated to the first author, Mr. M.M. Hedayat, an excellent M.Sc student at our Department, who passed away after finishing a major portion of the work. May God bless his soul. The authors would also like to thank the reviewers for their constructive comments.

\section{Appendix A: Derivation of the Viscosity Function in Pinho Model (Eq. 3)}

For completeness, this appendix provides some relationships in relation to the viscosity function as given by Eq. 3 (Ref. 34):

$$
\left.\begin{array}{l}
\mathrm{II}_{\mathrm{S}}=\frac{1}{2}\left[(\operatorname{trd})^{2}-\operatorname{trd}^{2}\right] \\
\text { for incompressible flow : } \operatorname{tr} \mathbf{d}=0
\end{array}\right\} \Rightarrow \mathrm{II}_{\mathrm{S}}=-\frac{1}{2} \operatorname{tr} \mathbf{d}^{2}
$$

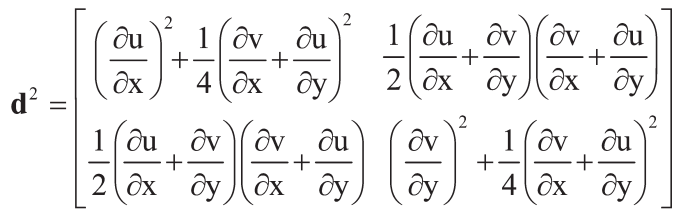

$$
\begin{aligned}
& \operatorname{trd}^{2}=\left(\frac{\partial u}{\partial x}\right)^{2}+\left(\frac{\partial v}{\partial y}\right)^{2}+\frac{1}{2}\left(\frac{\partial u}{\partial y}+\frac{\partial v}{\partial x}\right)^{2} \\
& \therefore \dot{\gamma}^{2}=-4 \mathrm{II}_{\mathrm{d}}=-4\left(-\frac{1}{2} \operatorname{trd}^{2}\right)=2 \operatorname{trd}^{2} \\
& =2\left(\frac{\partial u}{\partial x}\right)^{2}+2\left(\frac{\partial v}{\partial y}\right)^{2}+\left(\frac{\partial u}{\partial y}+\frac{\partial v}{\partial x}\right)^{2}
\end{aligned}
$$

$$
\begin{aligned}
& \operatorname{det} \mathbf{d}=\frac{\partial \mathrm{u}}{\partial \mathrm{x}} \cdot \frac{\partial \mathrm{v}}{\partial \mathrm{y}}-\frac{1}{4}\left(\frac{\partial \mathrm{u}}{\partial \mathrm{y}}+\frac{\partial \mathrm{v}}{\partial \mathrm{x}}\right)^{2} \\
& \therefore \dot{\varepsilon}=\frac{6 \operatorname{det} \mathbf{d}}{\operatorname{trd}^{2}}=6 \times \frac{\frac{\partial \mathrm{u}}{\partial \mathrm{x}} \cdot \frac{\partial \mathrm{v}}{\partial \mathrm{y}}-\frac{1}{4}\left(\frac{\partial \mathrm{u}}{\partial \mathrm{y}}+\frac{\partial \mathrm{v}}{\partial \mathrm{x}}\right)^{2}}{\left(\frac{\partial \mathrm{u}}{\partial \mathrm{x}}\right)^{2}+\left(\frac{\partial \mathrm{v}}{\partial \mathrm{y}}\right)^{2}+\frac{1}{2}\left(\frac{\partial \mathrm{u}}{\partial \mathrm{y}}+\frac{\partial \mathrm{v}}{\partial \mathrm{x}}\right)^{2}}
\end{aligned}
$$

Thus, the viscosity equation (Eq. 3) can be obtained as:

$$
\begin{aligned}
\mu(\dot{\gamma}, \dot{\varepsilon})= & \mathrm{K}_{\mathrm{v}}|\dot{\gamma}|^{(\mathrm{n}-1)} \mathrm{K}_{\mathrm{e}}|\dot{\varepsilon}|^{(\mathrm{p}-1)} \\
= & \mathrm{K}_{\mathrm{v}} \mathrm{K}_{\mathrm{e}}\left[2\left(\frac{\partial \mathrm{u}}{\partial \mathrm{x}}\right)^{2}+2\left(\frac{\partial \mathrm{v}}{\partial \mathrm{y}}\right)^{2}+\left(\frac{\partial \mathrm{u}}{\partial \mathrm{y}}+\frac{\partial \mathrm{v}}{\partial \mathrm{x}}\right)^{2}\right]^{\frac{\mathrm{n}-1}{2}} \\
& \mid \begin{array}{l}
6 \times\left.\frac{\frac{\partial \mathrm{u}}{\partial \mathrm{x}} \cdot \frac{\partial \mathrm{v}}{\partial \mathrm{y}}-\frac{1}{4}\left(\frac{\partial \mathrm{u}}{\partial \mathrm{y}}+\frac{\partial \mathrm{v}}{\partial \mathrm{x}}\right)^{2}}{\left(\frac{\partial \mathrm{u}}{\partial \mathrm{x}}\right)^{2}+\left(\frac{\partial \mathrm{v}}{\partial \mathrm{y}}\right)^{2}+\frac{1}{2}\left(\frac{\partial \mathrm{u}}{\partial \mathrm{y}}+\frac{\partial \mathrm{v}}{\partial \mathrm{x}}\right)^{2}}\right|^{(\mathrm{p}-1)}
\end{array}
\end{aligned}
$$

\section{Appendix B: Flowchart of the LBM Code}

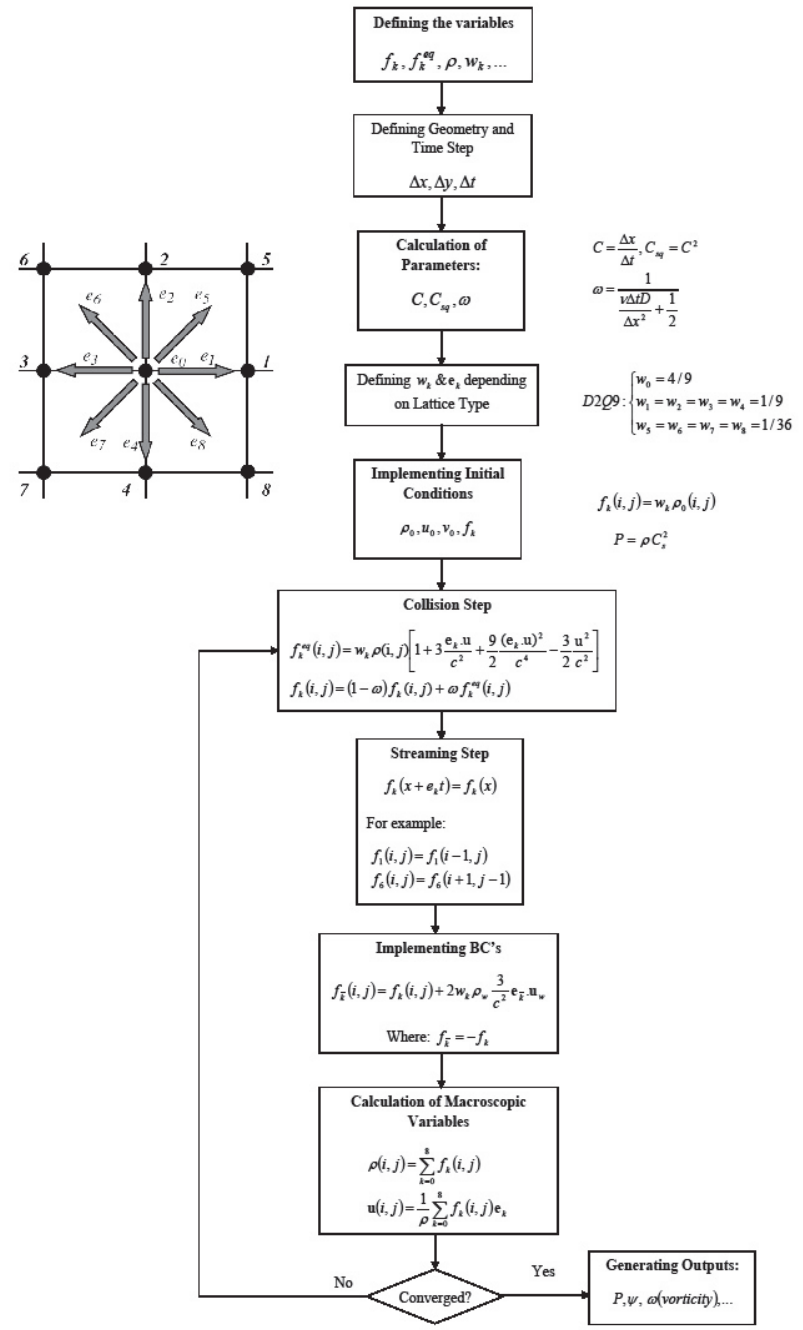




\section{REFERENCES}

1) Cochrane $T$, Walters $K$, Webster MF, On Newtonian and nonNewtonian flow in complex geometries, Phil Trans Soc Lond A, 301 (1981) 163-181.

2) Chen $S$, Doolen GD, Lattice Boltzmann method for fluid flows, Ann Rev Fluid Mech, 30 (1998) 329-364.

3) Ubertini $S$, Succi $S$, Recent advances of Lattice Boltzmann techniques on unstructured grids, Prog Comput Fluid Dyn, 5 (2005) 85-96.

4) Aharonov E, Rothman DH, Non-Newtonian flow (through porous media): A lattice Boltzmann method, Geophys Res Lett, 20 (1993) 679-682.

5) Rakotomalala N, Salin D, Watzky P, Simulations of viscous flows of complex fluids with a Bhatnagar, Gross, and Krook lattice gas, Phys Fluids, 8 (1996) 3200-3202.

6) Giraud L, d'Humi 'eres D, Lallemand P, A lattice Boltzmann model for viscoelasticity, Int J Mod Phys, C8 (1997) 805-815.

7) Ispolatov I, Grant M, Lattice Boltzmann method for viscoelastic fluids, Phys Rev, E 65 (2002) 056704.

8) Ginzburg I, Steiner K, A free-surface lattice Boltzmann method for modelling the filling of expanding cavities by Bingham fluids, Phil Trans R Soc Lond, A360 (2002) 453-466.

9) Lallemand P, d'Humi 'eres D, Luo L-S, Rubinstein R, Theory of the lattice Boltzmann equation: Three-dimensional model for linear visco-elastic fluids, Phys Rev, E67 (2003) 021203.

10) Boek ES, Chin J, Coveney PV, Lattice Boltzmann simulation of the flow of non-Newtonian fluids in porous media, Int $J$ Mod Phys, B17 (2003) 99-102.

11) Kehrwald D, Lattice Boltzmann Simulation of Shear-Thinning Fluids, J Stat Phys, 121 (2005) 223-237.

12) Sullivan SP, Gladden LF, Johns ML, Simulation of powerlaw fluid flow through porous media using lattice Boltzmann techniques, J Non-Newtonian Fluid Mech, 133 (2006) 91-98.

13) Boyd J, Buick J, Green S, A second-order accurate lattice Boltzmann non-Newtonian flow model, $J$ Phys A: Math Gen, 39 (2006) 14241-14247.

14) Sullivan SP, Sederman AJ, Johns ML, Gladden LF, Verification of shear-thinning LB simulations in complex geometries, $J$ Non-Newtonian Fluid Mech, 143 (2007) 59-63.

15) Psihogios J, Kainourgiakis ME, Yiotis AG, Papaioannou ATh, Stubos AK, A Lattice Boltzmann study of non-Newtonian flow in digitally reconstructed porous domains, Transp Porous Med, 70 (2007) 279-292.

16) Boyd J, Buick JM, Comparison of Newtonian and nonNewtonian flows in a two-dimensional carotid artery model using the lattice Boltzmann method, Phys Med Biol, 52 (2007) 6215-6228.

17) Boyd J, Buick JM, Green S, Analysis of the Casson and Carreau-Yasuda non-Newtonian blood models in steady and oscillatory flows using the lattice Boltzmann method, Phys Fluids, 19 (2007) 093103.
18) Yoshino M, Hotta Y, Hirozane T, Endo M, A numerical method for incompressible non-Newtonian fluid, $J$ Non-Newtonian Fluid Mech, 147 (2007) 69-78.

19) Wang $\mathrm{CH}$, Ho JR, Lattice Boltzmann modeling of Bingham plastics, Physica, A 387 (2008) 4740-4748.

20) Buick JM, Lattice Boltzmann simulation of power-law fluid flow in the mixing section of a single-screw extruder, Chem Eng Sci, 64 (2009) 52-58.

21) Wang D, Bernsdorf J, Lattice Boltzmann simulation of steady non-Newtonian blood flow in a $3 \mathrm{D}$ generic stenosis case, Computers and Mathematics with Applications (2009), (doi:10.1016/j.camwa.2009.02.020).

22) Ashrafizaadeh M, Bakhshaei H, A comparison of nonNewtonian models for lattice Boltzmann blood flow simulations, Computers and Mathematics with Applications (2009), (doi:10.1016/j.camwa.2009.02.021).

23) Gabbanelli S, Drazer G, Koplik J, Lattice Boltzmann method for non-Newtonian (power-law) fluids, Phys Rev, E72 (2005) 046312.

24) Agarwal RK, Chusak L, Morgan B, Lattice Boltzmann Simulations of Slip Flow of Newtonian and Non-Newtonian Fluids in Microgeometries, American Institute of Aeronautics and Astronautics, Paper 2009-1119 (2009).

25) Derksen JJ, Prashant, Simulations of complex flow of thixotropic liquids, J Non-Newtonian Fluid Mech, 160 (2009) 65-75.

26) Ghia U, Ghia KN, Shin CT, High-Re solutions for incompressible flow using the Navier-Stokes equations and a multigrid method, J Comp Phys, 48 (1982) 387-411.

27) Leong CW, Ottino GM, Increase in regularity by polymer addition during chaotic mixing in two-dimensional flows, Phys Rev Lett, 64 (1990) 874-877.

28) Pakdel P, McKinley GH, Cavity flows of elastic liquids: purely elastic instabilities, Phys Fluids, 10 (1998) 1058-1070.

29) Grillet AM, Shaqfeh ESG, Observations of viscoelastic instabilities in recirculation flows of Boger fluids, $J$ NonNewtonian Fluid Mech, 64 (1996) 141-155.

30) Bird RB, Armstrong RC, Hassager O, Dynamics of Polymeric Liquids, Vol. 1, 2nd Edition, John Wiley \& Sons, New York, 1997.

31) Pinho FT, A GNF framework for turbulent flow models of drag reducing fluids and proposal for a k- $\varepsilon$ type closure, $J$ NonNewtonian Fluid Mech, 114 (2003) 149-184.

32) Cruz DOA, Pinho FT, Turbulent pipe flow predictions with a low Reynolds number $\mathrm{k}-\varepsilon$ model for drag reducing fluids, J Non-Newtonian Fluid Mech, 114 (2003) 109-148.

33) Succi S, The Lattice Boltzmann Equation for Fluid Dynamics and Beyond, Clarondon Press, Oxford, 2001. 